\title{
Development of a Wearable Biomedical Heath-Care System
}

\author{
Han-Pang Huang ${ }^{1}$, and Lu-Pei Hsu ${ }^{2}$ \\ ${ }^{\prime}$ Correspondence, Professor of Department Mechanical Engineering, National Taiwan University, 106, Taipei, Taiwan \\ E-mail: hanpang@ntu.edu.tw, TEL/FAX: +886-2-23633875 \\ ${ }^{2}$ Graduate student of Graduate Department Mechanical Engineering, National Taiwan University, 106, Taipei, Taiwan
}

\begin{abstract}
This paper develops a wearable biomedical healthcare system which incorporates the wearable computing technology with multi-agent software architecture to provide a solution to the medical-care problems. The embedded Linux based wearable medical computer connects various sensors which can detect the patients' vital signs, such as electrocardiogram (ECG), body temperature, and body humidity. In order to get more reliable and accurate information, the multisensor fusion algorithm is employed to integrate the sensor information. The filter banks based QRS complex and heart beat detection algorithm, and fuzzy fusion algorithms are implemented to construct the diagnosis system of this wearable biomedical computer. Furthermore, the Personal Nursing Agent (PNA) and multi-agent based infrastructure for modern patient information and management system will be developed. The experimental results show that this system is helpful and useful for managing and monitoring patients. Accordingly, the wearable biomedical health-care system is regarded as a highly efficient biomedical system and inherits both features of multi-agent architecture and wearable computing, i.e., decentralized, reliable, autonomous, unrestrictive, and cooperative features.
\end{abstract}

Index Terms - wearable, electrocardiogram, embedded Linux, and PNA

\section{INTRODUCTION}

Since the twenty-first century, aging population is emerged as a preeminent worldwide phenomenon. The large proportion of elderly population leads to a series of medical problems, such as inadequate medical care resource, low quality of medical service. These incoming problems have strong impact on hospitals and nursing homes. It is getting more difficult to provide good health-care programs for those patients. Moreover, insufficient amounts of doctors and nurses can not monitor these large numbers of elderly people who have unstable health conditions. In order to solve these medical problems, this paper develops a wearable biomedical health-care system which integrates the wearable computing technology and multi-agent software architecture. This system improves the traditional bedside patient monitoring system that is only used in ICU (intensive care unit). The proposed biomedical system provides modularized functions and has the capability of monitoring mobile patients.

In the past, many researchers were focused on the development of home automation and wearable computing fields, but few of them tried to integrate these two fields. In this paper, home automation software architecture and wearable computing are integrated and applied to the medical institutions for improving their present medical health-care systems. Multi-agent architecture is adopted and implemented in this medical health-care system. The personal nursing agent concept is proposed. This agent serves as a personal nurse that monitors a patient's health condition 24-hour a day. The embedded Linux based system which wirelessly connects the patient information and management system is constructed. The vital signs acquisition modules are implemented, such as ECG acquisition module, body temperature sensing module, and body humidity sensing module. Multi-sensor fusion technology is applied to this biomedical health-care system. The filter bank based QRS detection algorithm is implemented to provide a high accuracy heart beat detection technique.

This paper is organized as follows. In section 2, an integrated architecture for health-care system is proposed. This system is used to improve the traditional bed-side patient monitoring and management system. The implementation and experimental results of the wearable biomedical health-care system will be presented in section 3 . Finally, conclusions are given in section 4 .

\section{INTEGRATED ARCHITECTURE FOR 24-HOUR HEALTH CARE SYSTEM}

Patient monitoring and management has been a research topic for years. As shown in Fig. 1, patient management and monitoring system can be divided into two major research components: patient monitoring system and patient management system. In general, the patient monitoring system is bedside medical monitoring system and only in charge of single patient. Meanwhile, the patient management system manages all hardware and software infrastructures in a building such as hospitals. The patient monitoring system is composed of four units: sensing units, information and monitoring system, life support facilities, and physicians. This monitoring system is a classical bedside medical monitoring instrument. The vital signs from the patient are sensed, amplified, filtered, and acquired. Afterward, these signals in the information fusion block are processed by algorithms that allow, in a somewhat limited manner, waveform detection and delineation. Events are also detected and alarms are then triggered in terms of different levels. These processed signals usually are displayed on a screen and useful information is 
stored in the database. Watrous described a neural network ECG monitoring algorithm to reduce the number of ventricular and normal beats classification errors [15]. Muhammad proposed an algorithm based on digital filtering, adaptive thresholding, statistical properties, and differencing of local max-min for real-time measurement of the fetal and maternal heart rates [5].

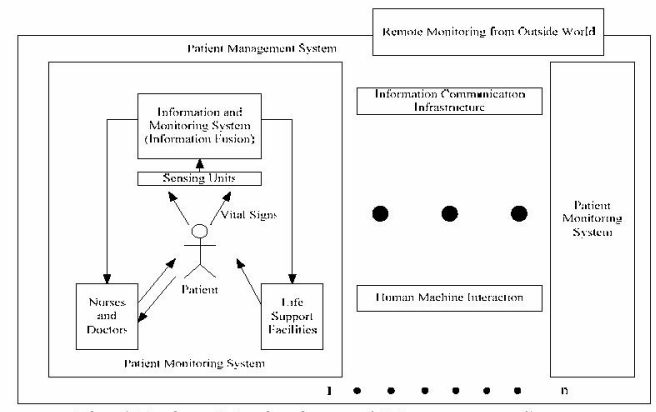

Fig. 1 Patient Monitoring and Management System

Although there are many relevant researches on the development of biomedical systems, they seldom address the whole architecture of patient monitoring and management. A review paper written by Mora reported the relevant works on patient monitoring and management systems [9]. It covers the traditional bedside patient monitoring, some medical information fusion flow and algorithms, and detailed information analysis. But the report does not mention about the whole architecture as depicted in Fig. 1. Compared with other researches, Varady [13] proposed an architecture for patient monitoring which focuses on the development of an industry standard based architecture to provide an open and standard system. The patient monitoring and management architecture described above is a typical concept that can meet most requirements in general medical institutes. However, the patient monitoring system in Fig. 1 is a bedside monitoring system and is useful only when patients lie on the bed. Emergency might occur while patients are not lying on the bed. In other words, the previous architecture is not suitable for monitoring "moving" patients. Moreover, the management functionality of that architecture will be failed if most of patients do not lie on the bed all day. Obviously, the management system is not working in this case. As a result, the ability of mobility management should be one of the main concerns in the modern patient monitoring and management system.

\section{A. Multi-Agent Architecture for Health-Care System}

In order to provide a safe and convenient environment for patients who live in hospitals and nursing homes, the patient monitoring and management system should have the following features:

- Full-time health condition tracking: Some patients might break out sometimes because of their unstable physical conditions. These unpredictable accidents may cause sudden death. The system should inform medical personnel immediately when the accidents occur.
- Reliability: This system manages important facilities in the building. It can not crash due to failure occurred in the sub-system.

- Autonomous: Due to limited number of nurses, the system should give a preliminary diagnosis to help nurses and doctors monitor the health conditions of patients.

The multi-agent system is a highly efficient system which provides reliability and extensibility in an automation system. Thus, this paper adopts the multi-agent architecture to fulfill the needs of patient information and management system. Online monitoring of the patients' conditions is the most important characteristics in the health-care system. In the proposed architecture, it also provides a mobile monitoring device which is worn on the patient. This wearable device can gather the vital signs, which are ECG signal, heart rate, body temperature, and body humidity. Medical personnel can monitor and act appropriately according to these vital signs. The overview of the proposed architecture is shown in Fig. 2.

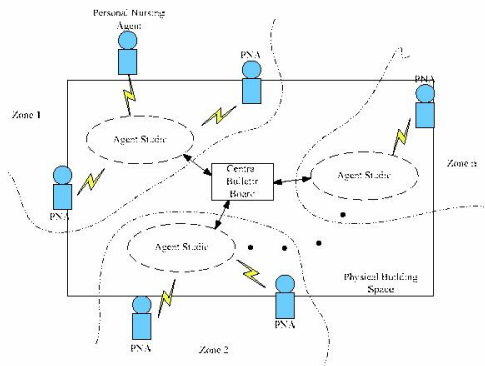

Fig. 2 Overview of Proposed Architecture

The proposed system consists of three major components: personal nursing agent (PNA), agent studio and central bulletin board, as shown in the

. The system can be further divided into several zones. Each zone represents an independent working group and can communicate with each other via the central bulletin board. The central bulletin board is a place where every component of the system announces its messages and records important system information such as alarm messages. A zone consists of an agent studio and several personal nursing agents and works as a small multi-agent system. In other words, an individual zone is a complete system and can work well without others. In the hospital which adopts this integrated architecture, generally, a zone represents an individual room and is regarded as a control space. A patient who lives in the hospital can wear a mobile device which is a part of personal nursing agent. The personal nursing agent carries the patient's personal information such as ID and password.

\section{B. Collaboration between Personal Nursing Agents and Agent Studio}

Each room is treated as a zone in the proposed architecture and each personal nursing agent represents a patient in the room, as shown in Fig. 3 and Fig. 4. The agent studio manages room devices and personal nursing agents. It communicates with the personal nursing agents through IEEE $802.11 \mathrm{~b}$ wireless network protocol. The agent studio can turn 
on and off the lights and adjust the air conditioner to the desired temperature when the agent studio receives a control command either from personal nursing agent or from the agent outside the zone. Once personal nursing agent sends the register command to the agent studio, the agent studio checks the ID and password sent from the personal nursing agent. If registration is successful, the agent studio will send the registration information to the central bulletin board.

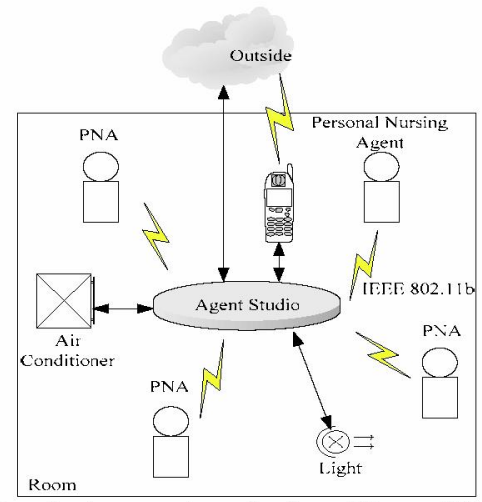

Fig. 3 Collaboration between Agent Studio and Personal Nursing Agents

\section{Collaboration between Agent Studios}

In this proposed architecture, every message sent by an agent will be recorded on the central bulletin board. In addition, doctors can post their messages and commands on the central bulletin board, and then the command will be sent to the agent.

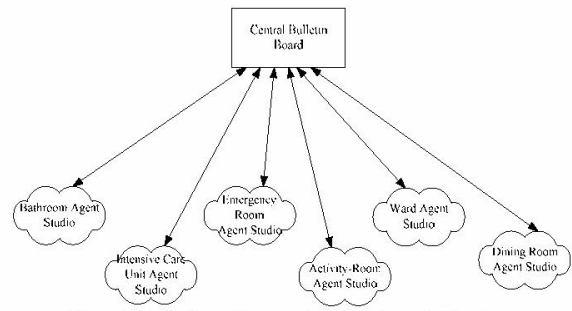

Fig. 4 Collaboration between Agent Studios

D. Multi-Sensor Fusion and Integration for Biomedical Health-Care System

In this biomedical health-care system, multiple sensors are used to sense some useful vital signs. These vital signs are used to preliminarily diagnose the patient condition and help doctors make decision. Fig. 5 shows the functional diagram of multi-sensor fusion and integration of this biomedical healthcare system. Multiple sensors sense the vital signs all the times, and vital signs will then be mixed together since only one single-chip processor is in charge of acquiring sensor signals. ECG signals need to be preprocessed order to filter out the noise. In the stage of mixed-signal separation, ECG signals are extracted from the mixed signal and sent to the fusion stage. Others are also separated out and interpreted to some meaningful values by interpreter. In the fusion stage, two algorithms are implemented to calculate the heart rate and make decisions to the action unit. The filter-banks QRS detection algorithm is used to detect the QRS complex in ECG diagram. QRS complex is an important wave for determining some useful heart conditions. Finally, the fuzzy diagnosis unit collects all information to determine the patient's physical condition.

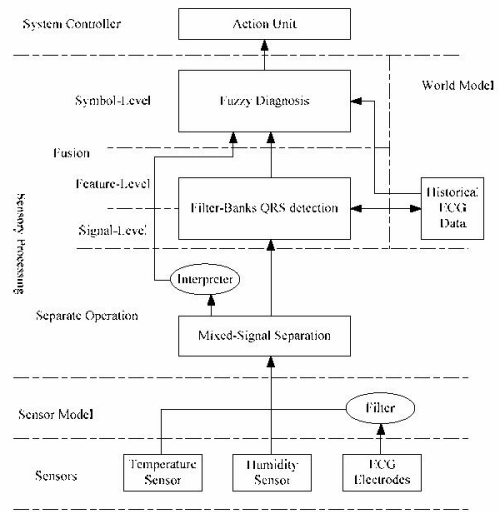

Fig. 5 Multi-Sensor Fusion and Integration of Biomedical Health-Care System

\section{E. Software System}

The detailed system components and the interaction between components of the proposed system are given in Fig. 6. Personal nursing agent is the lower-left block and consists of five components which are sensor data interpreter, fuzzybased diagnosis system, personal profile, communication management, and user agent. In this figure, the personal nursing agent and the agent studio are distinguished by different colors. The personal nursing agent is painted light blue and the other is painted light red. All components are working together on either a room gateway or a wearable computer.

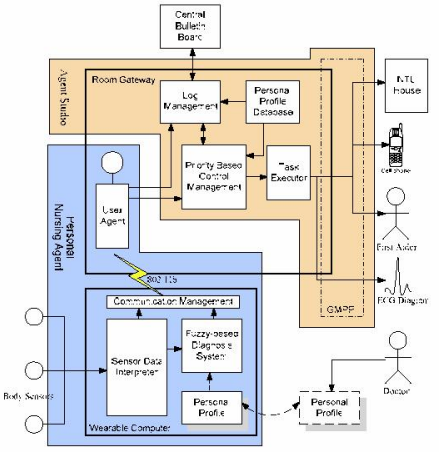

Fig. 6 Interaction and Detailed Components of System

\section{F. Sensor Data Interpreter}

The most important task of sensor data interpreter is to interpret the rough data to the useful data. There are several body sensors for sensing the vital signs of a patient simultaneously; and this will lead to the following problems:

- Mixed vital signs: The ECG sensor, body temperature sensor and humidity sensor sense the vital signs all the times. 
- Heart-rate calculation: The R-R interval of the ECG chart represents the period of heartbeat. Therefore, we can obtain the heart rate by reversing the period.

Fig. 7 shows the processing flow of the sensor data and the functionality of the sensor data interpreter. Three types of signals are mixed together before they are sent to the sensor data interpreter. After signals are processed by the sensor data interpreter, ECG signals will be separated and sent to the communication management. Heart rate will be calculated and sent to the communication management. Body temperature and humidity will be separated and sent to the communication management. The values of heart rate, temperature and humidity will be sent to the fuzzy based diagnosis system, and the patient condition should be diagnosed and current patient status will be determined.

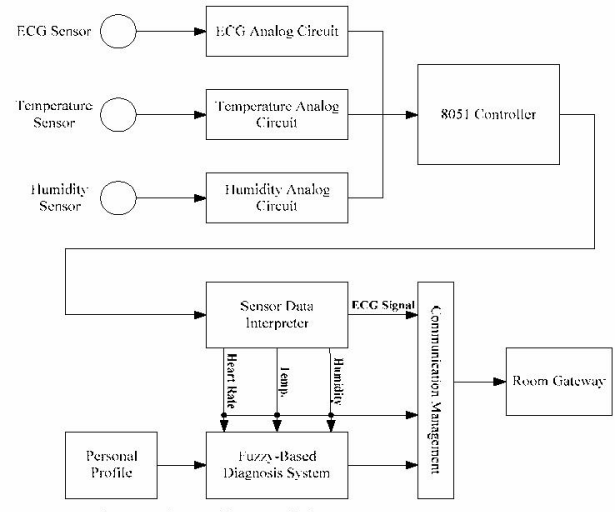

Fig. 7 Flow Chart of Sensor Data Interpreter

G. QRS Complex and Heart Beat Detection Algorithm

Detecting QRS complex in the ECG is one of the most important tasks. This stage is crucial in basic ECG monitoring systems and is important for all other ECG processing applications.

The ECG characteristic shape, shown in Fig. 8, is served as the basis for the automated determination of heart rate. QRS detection is difficult because of various types of noises in the ECG signal. Noise sources include power line interference, baseline wander, artifacts due to electrode motion, and muscle noise. Many detection algorithms have been proposed, for example, artificial neural networks [14], genetic algorithms [11], wavelet transforms [12], filter banks [1], and so on. Due to the real-time requirement of this system, the detection algorithm should be less computation load and high accuracy. Fortunately, most important algorithms have been made a detailed comparison by Kohler [6]. That research paper reports that different algorithm has different performance. Two parameters are used to evaluate the algorithms; that is,

$$
\begin{aligned}
& S e=\frac{T P}{T P+F N} \\
& +P=\frac{T P}{T P+F P}
\end{aligned}
$$$$
\text { Sensitivity... }
$$

Positive predictivity... where TP denotes the number of true positive detections, FN the number of false negatives, and FP the number of false positives.

The comparison results show that most of the algorithms have high sensitivity and positive predictivity more than $99 \%$ However, not all of them are tested against a standard database; that means not all the comparison results are reliable. This paper adopts a filter bank based algorithm proposed by Afonso [1]. In that comparison report, the filter bank based algorithm has both advantages of high accuracy and low computational load. The sensitivity and positive predictivity of the algorithm have the values greater than 99.5\%. Therefore, the filter bank based algorithm is suitable for the on-line QRS complex and heart beat detection techniques. Some relevant researches of the filter bank based QRS detection algorithm can be found in references $[2][3][4][10]$. A filter bank is an array of band-pass filters that span the entire audible frequency spectrum, see Fig. 9. The bank is used to isolate different frequency components in a signal. The amplitude response of the filter bank is shown in Fig. 9, where $w$ is the bandwidth of the filter. The $\mathrm{dB}$ is the amplitude of the system function and is referred to as the gain of the system. In order to facilitate the detection process, the ECG signal should be preprocessed prior to the filter-banks detection [10].

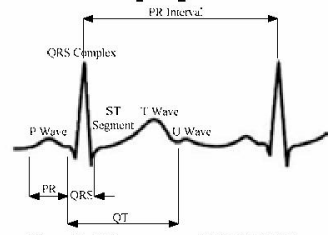

Fig. 8 Diagram of ECG Waves and Interval

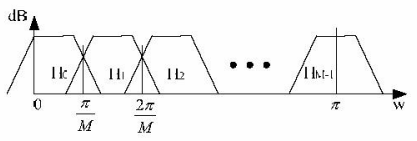

Fig. 9 Filter Banks

\section{H. Fuzzy-based Diagnosis System}

This health-care system has the ability to determine which patient is in dangerous by using fuzzy-based diagnosis system to preliminarily diagnose the patient's physical condition. This fuzzy-based diagnosis system diagnoses the patient's physical condition in terms of three input vital signs, heart rate, body temperature, and body humidity. A warning level which ranges from 0 to 10 corresponds to the three input vital signs, as shown in Fig. 10. The warning levels of the patient will then be grouped into three levels: normal level, noticed level, and dangerous level (Fig. 11).

As discussed in previous section, the arrhythmia might occur sometimes and cause sudden death. It can be detected by reading ECG. It is difficult to give an accurate diagnosis of arrhythmia, since it is related to not only detection algorithms but also well-understanding of domain knowledge. Thus, in addition to these three kinds of vital signs, sudden increase or decrease of heart rate can be used to diagnose the patient condition.

The basic architecture of a fuzzy decision logic system [7] is shown in Fig. 12, where $\mathrm{X}$ means the crisp measured data and $\mathrm{Y}$ the crisp output value, i.e., the input vital signs and output warning level, respectively. The fuzzy rule base stores 
the empirical knowledge of the process operation of the domain experts. Here, the domain experts will be doctors, and they have the responsibility to design different fuzzy rules for each patient.

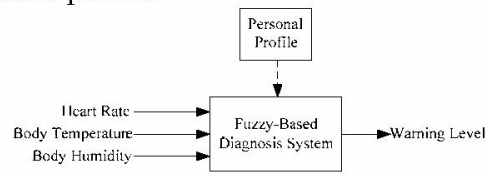

Fig. 10 Fuzzy-Based Diagnosis System

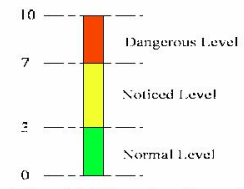

Fig. 11 Warning Level

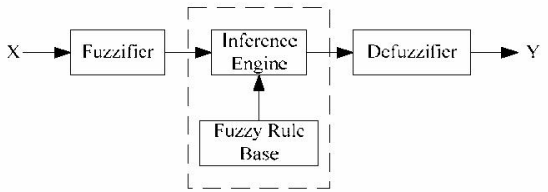

Fig. 12 Fuzzy Decision System

\section{Personal Profile}

The patient information and management system should have the ability to identify each patient inside building. Personal profile records personal information which provides the system to identify different persons. According to fuzzy theory [7], we can adjust the size of fuzzy partition for different patients. For example, Fig. 13 and Fig. 14 show two different membership functions for two different patients. The medium heart rate for patient $A$ ranges from 62 to 75 , meanwhile, the heart rate from 67 to 76 is the medium level for patient B. Since there are three input variables, there will be twenty-seven fuzzy rules in the fuzzy rule base.

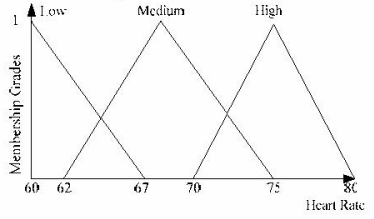

Fig. 13 Membership Function of Patient A

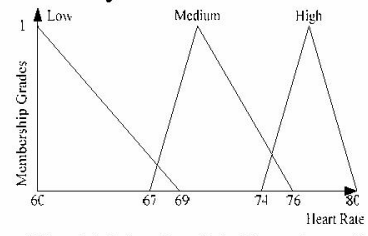

Fig. 14 Membership Function of Patient B
J. Priority Based Control Management

The biomedical health-care center is a big system that there are multiple components and many people coexist in the same space. In general, the following actions should be taken by the center:

- Emergency event: The alarm message is generated when a patient is diagnosed in a dangerous level.

- Regular control command: Sometimes the patients or doctors adjust the room devices to the desired state.

- Record patient's information: The patient's information, such as location and $\log$ time, will be recorded when they register.

- Plot ECG diagram: A doctor sometimes requests the system to plot a specific patient's ECG diagram for helping the doctor diagnose the patient.

- Transmit requested data to another Agent Studio: The doctor who requests system to plot ECG diagram might not be in the same zone as the patient. In this case, the ECG data should be forwarded to another Agent Studio.
The priority based control management which is a preemptive scheduling system schedules tasks according to their priorities. Accordingly, the control management will schedule all tasks according to the priorities of tasks, as shown in Fig. 15. Furthermore, if multiple task requests are sent to the control management at the same time, the tasks will be rescheduled according to the users' priority, as shown in Fig. 16 .

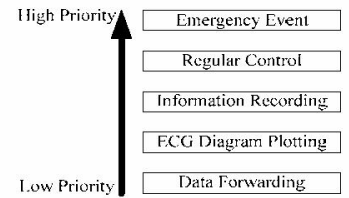

Fig. 15 Priorities of Tasks

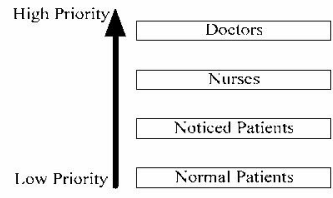

Fig. 16 Priorities of Users

\section{K. Log Management and Personal Profile Database}

The main job of $\log$ management is to verify the ID and password of the users. All patients' information will be recorded in the personal profile database including ID, password, priority, and so on. Consequently, the user can be identified and priority based control management can then receive the user's request message. After registration is successful, log management will record the user's log time and $\log$ location. The log location is the room gateway's IP that the patient can be located and the patient's vital sign data can be forwarded to another Agent Studio.

\section{Hardware System - ECG Acquisition Module}

The electrocardiogram (ECG) is a graphic recording of the changes occurring in the electrical potentials between different locations on the skin (leads) as a result of cardiac activity. In practice, this flow of electricity can also be sensed by recording electrodes [16]. However, it is difficult to record the ECG due to the low frequency and low amplitude of the electrical activity signal of heart. In general, the ECG signal frequency ranges from $0.05 \mathrm{~Hz}$ to $100 \mathrm{~Hz}$ and the amplitude ranges from $1 \mathrm{mV}$ to $10 \mathrm{mV}$. There are two predominant types of noises that contaminate the ECG signal acquired: the baseline wander (BW) noise and electrode motion artifact, and electromyogram-induced noise (EMG). The frequency components of $\mathrm{BW}$ noise are usually below $0.5 \mathrm{~Hz}$. EMG noise is dominant at higher frequencies, caused by increased muscle activity and by mechanical forces acting on the electrodes [8]. Therefore, the original ECG signal has to be amplified and filtered in order to be accurately processed.

\section{III.EXPERIMENTS AND VERIFICATIONS}

\section{A. System Implementation Overview}

The implementation overview is shown in Fig. 17, and the Agent Studio is implemented in a Win 2000 server with a database. The wearable computer is implemented in a handheld computer with embedded Linux OS. The filterbanks based QRS complex detection algorithm and Fuzzy diagnosis system are implemented on the hanldheld computer. The vital signs acquisition module is implemented 
in a circuit board and connected to the handheld computer by RS232 serial communication. All programs of wearable computer are developed on Embedded Linux Development Server, and then cross-compiled to the StrongARM based handheld computer.

Since this paper is focused on the development of software architecture and wearable biomedical computer, a model house called NTU-House is used to simulate the real building appliances and devices. The cell phone is used to send alarm messages to the person outside the building and the alarm messages are also sent to relevant persons by e-mail. Fig. 18 show the picture of the wearable biomedical system.

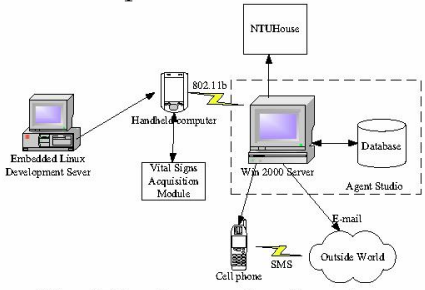

Fig. 17 Implementation Overview

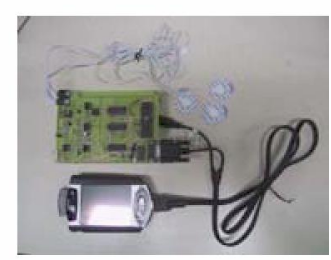

Fig. 18 Wearable Biomedical System

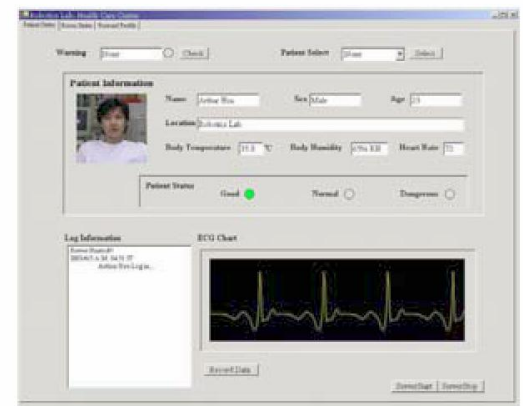

Fig. 19 User Interface of Patient Status

\section{B. Patient Status}

Fig. 19 shows the user interface of the patient status. The central block named Patient Information shows the patient's current condition, including name, sex, age, current location, body temperature, body humidity, heart rate, picture, and the result of preliminary diagnosis. Doctors can easily get the patient current condition from this user interface. We can see the current condition of the patient in the picture is "Good" and the light is green. If the patient's condition is dangerous, then the color of the light in Dangerous will be red. Nurses then can quickly find out the patient according to the patient's current location. The bottom right block named ECG Chart shows the current ECG of the patient, and the ECG data can be recorded by clicking the Record Data button for off-line analysis. The bottom left block named Log Information shows the log information of patients in this room.

\section{IV.CONCLUSIONS}

Compared with the traditional bedside patient monitoring and management system, this wearable biomedical system provides more flexible and more powerful abilities to monitor and manage the patients in modern hospitals and nursing homes. In this system, the multi-agent software architecture, filter-banks based QRS detection algorithm, multi-sensor fusion technology, and wearable computing are implemented. This system also proves that the multi-agent architecture is feasible in medical system and filter-banks based QRS detection algorithm is a highly efficient and reliable algorithm for heart beat detection.

Because of the multi-agent architecture, this system does not suffer from a single point failure associating with the centralized system. Each agent has its decision system to act automatically. Each component is independent to each other, thus, it is effortless to add a newly agent to the system. Agents of this system can cooperate with each other to accomplish a common task. Because of the wireless communication ability, every patient can do other things while wearing this wearable biomedical system. Each patient's location can be tracked easily. The embedded system has several sensors which perceive the body status. This ultimately increases situational awareness.

\section{REFERENCES}

[1] V. X. Afonso, W. J. Tompkins, T.Q. Nguyen, Shen Luo, "ECG beat detection using filter banks," IEEE Transactions on Biomedical Engineering, Vol. 46, issue 2, pp. 192-202, Feb. 1999.

[2] V. X. Afonso, W. J. Tompkins, T.Q. Nguyen, Shen Luo, "Comparing stress ECG enhancement algorithms," IEEE Engineering in Medicine and Biology Magazine, Vol. 5, issue 3, May-Jun. 1996.

[3] V. X. Afonso, O. Wjeben, W. J. Tompkins, T.Q. Nguyen, Shen Luo, "Filter bank-based ECG beat classification," IEEE International Conference on Engineering in Medicine and Biology society, Vol. 1, pp.30, Oct.-Nov. 1997.

[4] V. X. Afonso, W. J. Tompkins, T.Q. Nguyen, Shen Luo, "Multirate processing of the ECG using filter banks," IEEE Computers in Cardiology, pp.245-248, Sep. 1996.

[5] M.I Ibrahimy, F. Ahmed, M.A.M. Ali, E. Zahedi, "Real-time signal processing for fetal heart rate monitoring," IEEE Transactions on Biomedical Engineering, Vol. 50, issue 2, pp.258-261, Feb. 2003.

[6] B.-U., Kohler, C., Hennig, R., Orglmeister, "The principles of software QRS detection," IEEE Engineering in Medicine and Biology Magazine, Vol. 21, issue 1, pp. 42-57, Jan.-Feb. 2002.

[7] Chin-Teng Lin, C.S. George Lee, Neural Fuzzy Systems, $1^{\text {st }}$ Edition, Singapore: Prentice-Hall Pte Inc., pp. 142-166, 1996.

[8] GB Moody, WK Muldrow, and RG Mark, "A noise stress test for arrhythmia detectors," Computers in Cardiology, pp.381-384, 1984.

[9] F.A Mora, G. Passariello, G. Carrault, J.-P Le Pichon, "Intelligent patient monitoring and management systems: a review," IEEE Engineering in Medicine and Biology Magazine, Vol. 12, issue 4, pp.23-33, Dec. 1993.

[10] J. Pan and W. J. Tompkins, "A real-time QRS detection algorithm," IEEE Transactions on Biomedical Engineering, Vol. 3, pp.230-236, 1985.

[11] R. Poli, S. Cagnoni, and G. Valli, "Genetic design of optimum linear and nonlinear QRS detectors," IEEE Trans. Biomed. Eng., Vol. 42, pp.1137$1141,1995$.

[12] G. Strang and T. Nguyen, Wavelets and Filter Banks, Cambridge, MA: Wellesley-Cam-bridge Press, 1996.

[13] P. Varady, Z. Benyo B., Benyo, "An open architecture patient monitoring system using standard technologies," IEEE Transactions on Information Technology in Biomedicine, Vol.6, issue 1, Mar. 2002.

[14] G. Vijaya, V. Kumar, and H.K. Verma, "ANN-based QRS-complex analysis of ECG," J. Med. Eng. Technol., Vol. 22, Issue 4, pp. 160-167, 1998.

[15] R. Watrous, G Towell, "A patient-adaptive neural network ECG patient monitoring algorithm," IEEE Computers in Cardiology, pp.229-232, Sep. 1995.

[16] J.G., Webster, Medical Instrumentation: Application and Design, 3rd Edition, John Wiley \& Sons, Inc., 1998. 